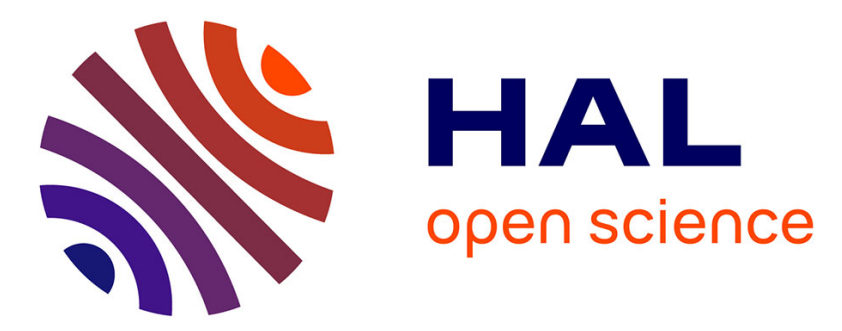

\title{
Lyapounov exponents of products of random matrices: weak disorder expansion. - Application to localisation
}

\author{
B. Derrida, K. Mecheri, J.L. Pichard
}

\section{To cite this version:}

B. Derrida, K. Mecheri, J.L. Pichard. Lyapounov exponents of products of random matrices: weak disorder expansion. - Application to localisation. Journal de Physique, 1987, 48 (5), pp.733-740. 10.1051/jphys:01987004805073300 . jpa-00210492

\section{HAL Id: jpa-00210492 https://hal.science/jpa-00210492}

Submitted on 1 Jan 1987

HAL is a multi-disciplinary open access archive for the deposit and dissemination of scientific research documents, whether they are published or not. The documents may come from teaching and research institutions in France or abroad, or from public or private research centers.
L'archive ouverte pluridisciplinaire HAL, est destinée au dépôt et à la diffusion de documents scientifiques de niveau recherche, publiés ou non, émanant des établissements d'enseignement et de recherche français ou étrangers, des laboratoires publics ou privés. 


\title{
Lyapounov exponents of products of random matrices : weak disorder expansion. Application to localisation
}

\author{
B. Derrida, K. Mecheri $\left(^{+}\right)$and J. L. Pichard (*) \\ Service de Physique Théorique, CEN-Saclay, 91191 Gif sur Yvette Cedex, France \\ $\left(^{+}\right)$Laboratoire de Physique Statistique, Université des Sciences et de la Technologie Houari Boumediene, \\ Dar El Beida, BP 9 Alger, Algérie \\ (*) Service de Physique du Solide et de Résonance Magnétique, CEN-Saclay, 91191 Gif sur Yvette Cedex, \\ France
}

(Reçu le 7 octobre 1986, accepté le 18 décembre 1986)

\begin{abstract}
Résumé. - Nous donnons le développement de faible désordre des exposants de Lyapounov d'un produit de matrices aléatoires. Ce développement est valide quand les valeurs propres de la matrice en l'absence de désordre ont toutes des modules différents. En illustration, nous étudions la localisation des électrons sur des rubans dans la limite du faible désordre. Nous montrons que notre développement est en bon accord avec des calculs numériques quand la condition sur les modules est satisfaite, ce qui correspond à des énergies en dehors de la bande de conduction du système pur. Pour ces énergies, nous obtenons une densité limite des exposants de Lyapounov quand la largeur du ruban augmente. A l'intérieur de la bande, notre développement n'est valide que si une partie imaginaire est ajoutée à l'énergie.
\end{abstract}

Abstract. - We derive the weak disorder expansion of the Lyapounov exponents of a product of random matrices. The condition for this expansion to be valid is that in the limit of zero disorder, the matrix has all its eigenvalues with different moduli. As an example we study the problem of localisation on strips in the limit of weak disorder. We show that our expansion agrees very well with numerical simulations in the region where the condition on the moduli is satisfied which corresponds to energies outside the conduction band. In that region, we find a limiting density of Lyapounov exponents when the strip width goes to infinity. Inside the band, our expansion cannot be valid unless one adds an imaginary part to the energy.

\section{Introduction.}

Products of random matrices are very often used in the study one dimensional disordered systems (spins with random interactions or in random fields, electrons in a random potential, etc.) [1-3]. When one considers infinite products of random matrices, the first quantities which can be calculated are the Lyapounov exponents. Usually, the physical properties (free energy, magnetisation, density of states) are easy to obtain when the Lyapounov exponents are known. Unfortunately there does not exist any general method which gives the expression of the Lyapounov exponents.

In the simplest cases (one dimensional models with nearest neighbour interactions), the random matrices are $2 \times 2$ matrices and there exists a few methods to expand the Lyapounov exponents in various limiting cases. In particular, one knows how to make weak disorder expansions when the random matrices have small fluctuations around their average value [3-7].

When one tries to study more complex situations like disordered chains having longer range interactions than nearest neighbours or disordered systems on strips, one has to consider infinite products of random matrices of size $n>2$. For magnetic systems, the free energy is given by the largest Lyapounov exponent whereas the second Lyapounov exponent is needed to compute the length which characterizes the decrease of correlation functions. In the localisation problem, it is the smallest positive Lyapounov exponent which gives the localisation length [8-10]. So in general, the study of a disordered system 
requires the calculation of not only the largest Lyapounov exponent but of all of them. One should notice also that several recent works on disordered systems or on dynamical systems have shown the importance of the whole distribution of Lyapounov exponents [11-15].

The purpose of the present work is to give a perturbative expansion of the Lyapounov exponents of products of random matrices in the limit of weak disorder.

In section 2, we derive this weak disorder expansion up to 4 th order. The conditions for this expansion to be valid is that in the unperturbed problem (i.e. in the limit of zero disorder), the random matrices have all their eigenvalues with different moduli.

In section 3, we consider the example of the localisation problem on strips of finite width. We write the expression of the random matrices and we use the results of section 2 to obtain the Lyapounov exponents.

In section 4, we compare the expressions obtained in 3 with results of numerical simulations.

\section{Weak disorder expansion of the Lyapounov ex- ponents.}

In this section we are going to derive the weak disorder expansion of the Lyapounov exponents of a product of random matrices :

$$
P=\prod_{\alpha=1}^{N} M_{\alpha} .
$$

The matrices $M_{\alpha}$ are $n \times n$ matrices and by weak disorder we mean that the $M_{\alpha}$ have the following form

$$
M_{\alpha}=A+\mu B_{\alpha}
$$

where $A$ is a fixed matric (independent of $\alpha$ ) and $B_{\alpha}$ is a random matrix. The matrices $A$ and $B_{\alpha}$ can have real as well as complex elements and need not have any symmetry. We only assume that the average of $B_{\alpha}$ vanishes

$$
\bar{B}_{\alpha}=0 \text {. }
$$

If (3) is not satisfied, one can always reduce the problem to a case where (3) is verified by including the average of $B_{\alpha}$ in $A$.

In order to obtain the weak disorder expansion of the Lyapounov exponents, we need to set the following condition on $A$ :

The $n$ eigenvalues of $A$ have all their moduli different .

We will see later why we need this condition. Let us just mention here that it is similar to perturbation theories where one needs to treat the degenerate case separately.

Because of (4), one can always work in the basis where $A$ is diagonal. Therefore we shall make all our calculations assuming that $A$ has the following diagonal form

$$
A=\left(\begin{array}{llll}
\lambda_{1} & & & 0 \\
& \lambda_{2} & \\
0 & & \ddots & \\
& & \lambda_{n}
\end{array}\right)
$$

with

$$
\left|\lambda_{1}\right|>\left|\lambda_{2}\right|>\left|\lambda_{3}\right|>\cdots>\left|\lambda_{n}\right|
$$

If one calls $\gamma_{p}$ the $p$-th Lyapounov exponent, our final result will be

$$
\begin{aligned}
\gamma_{1}+\gamma_{2}+\cdots & +\gamma_{p}=\sum_{j=1}^{p} \log \lambda_{j}- \\
& -\frac{\mu^{2}}{2} \sum_{i=1}^{p} \sum_{j=1}^{p} \frac{\overline{B^{i j} B^{j i}}}{\lambda_{i} \lambda_{j}} \\
& +\frac{\mu^{3}}{3} \sum_{i=1}^{p} \sum_{j=1}^{p} \sum_{k=1}^{p} \frac{\overline{B^{i j} B^{j k} B^{k i}}}{\lambda_{i} \lambda_{j} \lambda_{k}} \\
& -\frac{\mu^{4}}{4} \sum_{i=1}^{p} \sum_{j=1}^{p} \sum_{k=1}^{p} \sum_{l=1}^{p} \frac{\overline{B^{i j} B^{j k} B^{k l} B^{l i}}}{\lambda_{i} \lambda_{j} \lambda_{k} \lambda_{l}} \\
& -\frac{\mu^{4}}{2} \sum_{i=1}^{p} \sum_{j=1}^{p} \sum_{r>p}^{n} \sum_{s>p}^{n} \frac{\overline{B^{i r} B^{j s}} \overline{B^{r j} B^{s i}}}{\lambda_{i} \lambda_{j}\left(\lambda_{i} \lambda_{j}-\lambda_{r} \lambda_{s}\right)} \\
& +\mu^{4} \sum_{i=1}^{p} \sum_{j=1}^{p} \sum_{k=1}^{p} \sum_{r>p}^{n} \frac{\frac{B^{i r} B^{k i}}{B^{r j} B^{j k}}}{\lambda_{j} \lambda_{k}\left(\lambda_{k}-\lambda_{r}\right)} \\
& +0\left(\mu^{5}\right)
\end{aligned}
$$

where $B^{i j}$ is the matrix element $i, j$ of the matrix $B_{\alpha}$ and the bar denotes the average over disorder. If complex numbers appear on the right hand side of (7), one has to take the real part of the result.

Let us show now how formula (7) can be derived and how it could be generalized.

Let us call $\mathbf{U}_{1}, \mathbf{U}_{2}, \ldots, \mathbf{U}_{p}, p$ randomly chosen vectors of dimension $n$ and let us define $\mathbf{w}_{1}, \mathbf{w}_{2}, \ldots, \mathbf{w}_{p}$ by

$$
\mathbf{W}_{i}=\left(\prod_{i=1}^{N} M_{\alpha}\right) \mathbf{U}_{i}
$$

The sum of the first $p$ Lyapounov exponents is given by the exponential growth of a $p$ dimensional volume generated by $p$ vectors $[16,17]$. One possible measure of the volume generated by $U_{1}, U_{2}, \ldots, U_{p}$ is $\operatorname{det}_{p}\left(\mathbf{U}_{1}, \mathbf{U}_{2}, \ldots, \mathbf{U}_{p}\right)$ defined as the determinant of the $p \times p$ matrix where the $i$-th column consists of the first $p$ components of the vector $U_{1}$. 
Therefore, the first $p$ Lyapounov exponents can be obtained from the following formula :

$$
\begin{aligned}
\gamma_{1}+ & \gamma_{2}+\cdots+\gamma_{p}= \\
& =\lim _{N \rightarrow \infty} \frac{1}{N} \log \left[\frac{\operatorname{det}_{p}\left(\mathbf{W}_{1}, \mathbf{W}_{2}, \ldots, \mathbf{W}_{p}\right)}{\operatorname{det}_{p}\left(\mathbf{U}_{1}, \mathbf{U}_{2}, \ldots, \mathbf{U}_{p}\right)}\right] .
\end{aligned}
$$

In order to compute the Lyapounov exponents $\gamma_{1}, \ldots, \gamma_{p}$, we are going to do first a perturbation expansion $N$ being finite and fixed and then take the limit $N \rightarrow \infty$.

If one wants to compute the Lyapounov exponents up to an order $\mu^{q}$, it is enough to expand $P$ up to order $\mu^{q / 2}$ because all the higher order terms will disappear when we will average over disorder. Here we are going to compute the Lyapounov exponents up to 4 th order in $\mu$ and therefore we need only to expand $P$ up to 2 nd order in $\mu$. One then finds

$$
P=\left[0+\mu C+\mu^{2} D\right] A^{N}+0\left(\mu^{3}\right)
$$

where the matrices $C$ and $D$ are defined by

$$
\begin{aligned}
& C=\sum_{\alpha=1}^{N} A^{\alpha-1} B_{\alpha} A^{-\alpha} \\
& D=\sum_{1 \leqslant \alpha<\beta \leqslant N} A^{\alpha-1} B_{\alpha} A^{\beta-\alpha-1} B_{\beta} A^{-\beta} .
\end{aligned}
$$

We have now to compute $\log \left[\operatorname{det}_{p}(P)\right]$. Using (10) and the fact that $A$ is diagonal, one has

$$
\begin{aligned}
\log \left[\operatorname{det}_{p}(P)\right] & =\log \left[\operatorname{det}_{p}\left(A^{N}\right)\right]+ \\
+ & \log \left[\operatorname{det}_{p}\left(\mathbb{U}+\mu C+\mu^{2} D\right)\right] .
\end{aligned}
$$

Using the fact that $\log (\operatorname{det} M)=\operatorname{tr}(\log M)$ for any matrix $M$, one can replace (13) by

$$
\begin{aligned}
\log \left[\operatorname{det}_{p}(P)\right]= & \operatorname{tr}\left(\log \tilde{A}^{N}\right)+ \\
& +\operatorname{tr}\left[\log \left(\tilde{\mathbb{Q}}+\mu \tilde{C}+\mu^{2} \tilde{D}\right)\right]
\end{aligned}
$$

where $\tilde{A}, \tilde{\nabla}, \tilde{C}, \tilde{D}$ are $p \times p$ matrices which are obtained from $A, \mathbb{}, C$ and $D$ by keeping the first $p$ rows and columns.

We can now expand (14) up to 4 th order in $\mu$ and one gets :

$\log \left[\operatorname{det}_{p}(P)\right]=\operatorname{tr}\left(\log \tilde{A}^{N}\right)+\mu \operatorname{tr} \tilde{C}+$

$$
\begin{aligned}
& +\mu^{2}\left[\operatorname{tr} \tilde{D}-\frac{1}{2} \operatorname{tr}\left(\tilde{C}^{2}\right)\right] \\
& +\mu^{3}\left[\frac{1}{3} \operatorname{tr}(\tilde{C})^{3}-\operatorname{tr}(\tilde{C} \tilde{D})\right] \\
& +\mu^{4}\left[-\frac{1}{4} \operatorname{tr}(\tilde{C})^{4}+\operatorname{tr}\left(\tilde{C}^{2} \tilde{D}\right)-\frac{1}{2} \operatorname{tr}(\tilde{D})^{2}\right] \\
& +0\left(\mu^{5}\right)
\end{aligned}
$$

We have now to find the limiting behaviour of (15) in the limit $N \rightarrow \infty$. One has of course

$$
\lim _{N \rightarrow \infty} \frac{1}{N} \operatorname{tr} \tilde{C}=0
$$

because of (3) whereas

$$
\lim _{N \rightarrow \infty} \frac{1}{N} \operatorname{tr} \tilde{D}=\lim _{N \rightarrow \infty} \frac{1}{N} \operatorname{tr}(\tilde{C} \tilde{D})=0
$$

because of (3) and of (6).

Then we have to compute the remaining terms. One sees easily that

$$
\begin{aligned}
& \lim _{N \rightarrow \infty} \frac{1}{N} \operatorname{tr}\left(\log \tilde{A}^{N}\right)=\sum_{i=1}^{p} \log \lambda_{i} \\
& \lim _{N \rightarrow \infty} \frac{1}{N} \operatorname{tr}\left(\tilde{C}^{2}\right)=\sum_{i=1}^{p} \sum_{j=1}^{p} \frac{\overline{B^{i j} B^{j i}}}{\lambda_{i} \lambda_{j}} \\
& \lim _{N \rightarrow \infty} \frac{1}{N} \operatorname{tr}\left(\tilde{C}^{3}\right)=\sum_{i=1}^{p} \sum_{j=1}^{p} \sum_{k=1}^{p} \frac{\overline{B^{i j} B^{j k} B^{k i}}}{\lambda_{i} \lambda_{j} \lambda_{k}}
\end{aligned}
$$

$$
\begin{aligned}
\lim _{N \rightarrow \infty} \frac{1}{N} \operatorname{tr}\left(\tilde{C}^{4}\right)=\sum_{i=1}^{p} \sum_{j=1}^{p} & \sum_{k=1}^{p} \sum_{l=1}^{p} \frac{\overline{B^{i j} B^{j k} B^{k l} B^{l i}}}{\lambda_{i} \lambda_{j} \lambda_{k} \lambda_{l}}+ \\
& +\lim _{N \rightarrow \infty} \frac{1}{N} \sum_{\alpha=1}^{N} \sum_{\beta=1}^{N} \sum_{i=1}^{p} \sum_{j=1}^{p} \sum_{k=1}^{p} \sum_{l=1}^{p} \frac{\overline{B^{i j} B^{j k}} \overline{B^{k l} B^{l i}}}{\lambda_{i} \lambda_{j} \lambda_{k} \lambda_{l}}\left(\frac{\lambda_{i}}{\lambda_{k}}\right)^{\alpha-\beta} \\
& +\frac{\overline{B^{i j} B^{k l}} \frac{\alpha \neq \beta}{\lambda_{i} \lambda_{j} \lambda_{k} \lambda_{l}}\left(\frac{\lambda_{i} \lambda_{k}}{\lambda_{j} \lambda_{l}}\right)^{\alpha-\beta}+\frac{\overline{B^{i j} B^{l i}} \overline{B^{j k} B^{k l}}}{\lambda_{i} \lambda_{j} \lambda_{k} \lambda_{l}}\left(\frac{\lambda_{l}}{\lambda_{j}}\right)^{\alpha-\beta}}{}
\end{aligned}
$$

$$
\begin{aligned}
& \lim _{N \rightarrow \infty} \frac{1}{N} \operatorname{tr}\left(\tilde{C}^{2} \tilde{D}\right)= \lim _{N \rightarrow \infty} \frac{1}{N} \sum_{1 \leqslant \alpha<\beta \leqslant N} \sum_{i=1}^{p} \sum_{j=1}^{p} \sum_{k=1}^{p} \sum_{r=1}^{n} \frac{\overline{B^{i j} B^{k r}} \overline{B^{j k} B^{r i}}}{\lambda_{i} \lambda_{j} \lambda_{k} \lambda_{r}}\left(\frac{\lambda_{k} \lambda_{i}}{\lambda_{r} \lambda_{j}}\right)^{\alpha-\beta}+ \\
&+\frac{\overline{B^{i j} B^{r i}} \overline{B^{j k} B^{k r}}}{\lambda_{i} \lambda_{j} \lambda_{k} \lambda_{r}}\left(\frac{\lambda_{j}}{\lambda_{r}}\right)^{\alpha-\beta} \\
& \lim _{N \rightarrow \infty} \frac{1}{N} \operatorname{tr}(\tilde{D})^{2}=\lim _{N \rightarrow \infty} \frac{1}{N} \sum_{1 \leqslant \alpha<\beta \leqslant N} \sum_{i=1}^{p} \sum_{j=1}^{p} \sum_{r=1}^{n} \sum_{s=1}^{n} \frac{\overline{B^{i r} B^{j s}} \overline{B^{r j} B^{s i}}}{\lambda_{i} \lambda_{j} \lambda_{r} \lambda_{s}}\left(\frac{\lambda_{i} \lambda_{j}}{\lambda_{r} \lambda_{s}}\right)^{\alpha-\beta} .
\end{aligned}
$$


When one adds all these terms in (15), there are some cancellations and one ends up with

$$
\begin{aligned}
& \gamma_{1}+\gamma_{2}+\cdots+\gamma_{p}=\sum_{i=1}^{p} \log \lambda_{i}-\frac{\mu^{2}}{2} \sum_{i=1}^{p} \sum_{j=1}^{p} \frac{\overline{B^{i j} B^{j i}}}{\lambda_{i} \lambda_{j}}+ \\
& +\frac{\mu^{3}}{3} \sum_{i=1}^{p} \sum_{j=1}^{p} \sum_{k=1}^{p} \frac{\overline{B^{i j} B^{j k} B^{k i}}}{\lambda_{i} \lambda_{j} \lambda_{k}}-\frac{\mu^{4}}{4} \sum_{i=1}^{p} \sum_{j=1}^{p} \sum_{k=1}^{p} \sum_{l=1}^{p} \frac{\overline{B^{i j} B^{j k} B^{k l} B^{l i}}}{\lambda_{i} \lambda_{j} \lambda_{k} \lambda_{l}} \\
& -\frac{\mu^{4}}{2} \sum_{i=1}^{p} \sum_{j=1}^{p} \sum_{r>p}^{n} \sum_{s>p}^{n} \frac{\overline{B^{i r} B^{j s}} \overline{B^{r j} B^{s i}}}{\lambda_{i} \lambda_{j} \lambda_{r} \lambda_{s}} \lim _{N \rightarrow \infty} \frac{1}{N} \sum_{1 \leqslant \alpha<\beta \leqslant N}\left(\frac{\lambda_{r} \lambda_{s}}{\lambda_{i} \lambda_{j}}\right)^{\beta-\alpha} \\
& +\mu^{4} \sum_{i=1}^{p} \sum_{j=1}^{p} \sum_{k=1}^{p} \sum_{r>p}^{n} \frac{\overline{B^{i j} B^{r i}} \overline{B^{j k} B^{k r}}}{\lambda_{i} \lambda_{j} \lambda_{k} \lambda_{r}} \lim _{N \rightarrow \infty} \frac{1}{N} \sum_{1 \leqslant \alpha<\beta \leqslant N}\left(\frac{\lambda_{r}}{\lambda_{j}}\right)^{\beta-\alpha} .
\end{aligned}
$$

We see now why the condition (6) is necessary. In (23), the sum over $\alpha$ and $\beta$ has a limit when $N \rightarrow \infty$ only if $\left|\lambda_{r}\right|<\left|\lambda_{j}\right|$ for $1 \leqslant j \leqslant p$ and $r>p$ and if $\left|\lambda_{r} \lambda_{s}\right|<\left|\lambda_{i} \lambda_{j}\right|$ for all $i, j \leqslant p$ and $r$ and $s>p$. In the limit $N \rightarrow \infty$, these sums reduce to geometrical series and one gets (7).

Condition (6) can be understood in the following way. In the weak disorder limit $(\mu \rightarrow 0)$, the Lyapounov exponents become the logarithms of the eigenvalues of the matrix $A$. The largest Lyapounov becomes the logarithm of the eigenvalue which has the largest modulus. The second becomes the logarithm of the second eigenvalue and so on. So the ordering (6) is right in the limit $\mu \rightarrow 0$ and one has to choose this order for our expansion to be valid.

We see that one could continue the weak disorder expansion to higher orders. One should also notice that if two eigenvalues have the same modulus, condition (6) cannot be satisfied by reordering the eigenvalues and our formula is not valid in that case. By looking at the expansion (7), we also see why condition (6) is necessary. At each order, we see differences of eigenvalues in the denominator and so condition (6) prevents the appearance of small denominators. Condition (6) is probably too strong and formula (7) remains probably true if $\left|\lambda_{i}\right|>$ $\left|\lambda_{j}\right|$ for $1 \leqslant i \leqslant p$ and $p+1 \leqslant j \leqslant n$.

\section{Localization on disordered strips.}

The transport properties of electrons on two dimensional disordered strips have been studied in the framework of a one electron theory. The usual model consists of a tight binding Hamiltonian with diagonal disorder. The wave function $\psi_{i, j}$ at the point $(i, j)$ of a square lattice satisfies :

$$
\begin{aligned}
& \psi_{1+i, j}+\psi_{1-i, j}+\psi_{i, j+1}+ \\
& +\psi_{i, j-1}+\mu V_{i, j} \psi_{i, j}=E \psi_{i, j}
\end{aligned}
$$

where $\mu V_{i, j}$ is the random potential on site $(i, j)$.

On a strip of finite width $M$, one has

$$
\begin{gathered}
1 \leqslant i \leqslant M \\
-\infty<j<\infty .
\end{gathered}
$$

If one knows the wave function $\psi_{i, j}$ on two consecutive columns (for example on columns 0 and 1) one can compute the wave function on any other column using a product of $2 M \times 2 M$ random matrices. If one defines the $2 M$ component $\mathbf{X}_{L}$ by

$$
\left.\begin{array}{ll}
\left(X_{L}\right)_{i}=\psi_{i, L+1} \\
\left(X_{L}\right)_{i+M}=\psi_{i, L}
\end{array}\right\} \text { for } 1 \leqslant i \leqslant M .
$$

Then from (24), it follows that

$$
\mathbf{X}_{L}=\left(\prod_{\alpha=1}^{L} M_{\alpha}\right) \mathbf{x}_{0}
$$

where the transfer matrix $M_{\alpha}$ is a $2 M \times 2 M$ matrix which can be written as

$$
M_{\alpha}=A+\mu B_{\alpha}
$$

where

$$
\begin{aligned}
A & =\left(\begin{array}{cc}
E \mathbb{}-T_{\mathrm{H}} & -\mathbb{\square} \\
\mathbb{0} & \mathbb{O}
\end{array}\right) \\
B_{\alpha} & =\left(\begin{array}{cc}
\mathbb{V}_{\alpha} & \mathbb{O} \\
\mathbb{O} & \mathbb{O}
\end{array}\right)
\end{aligned}
$$

$\checkmark$ denotes the $M \times M$ identity matrix, $\mathbb{O}$ denotes the $M \times M$ matrix whose all elements are $0, T_{\mathrm{H}}$ is a $M \times M$ matrix defined by

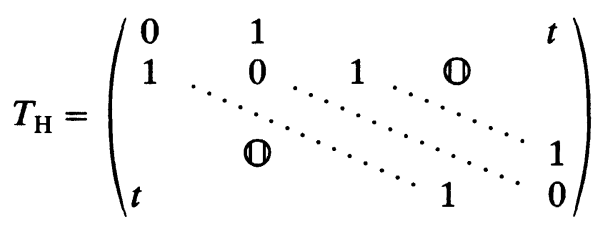

and $\mathbb{V}_{\alpha}$ is defined by

$$
\mathbb{V}_{\alpha}=\left(\begin{array}{ccc}
-V_{1, \alpha} & \mathbb{O} \\
\mathbb{O} & \ddots & \\
\mathbb{O} & & V_{M, \alpha}
\end{array}\right)
$$

The coefficient $t$ depends on the transverse boundary conditions on the strip. Periodic boundary conditions $\left(\psi_{i, j}=\psi_{i+M, j}\right)$ correspond to $t=1$. For rigid 
boundary conditions $\left(\psi_{0, j}=\psi_{M+1, j}=0\right)$ one should choose $t=0$. The parameter $t$ could also be a complex number in the presence of a transverse current or equal to -1 for antiperiodic boundary conditions.

Time reversal symmetry and current conservation imply that the transfer matrices $M_{\alpha}$ are symplectic. As a consequence, their eigenvalues are pairs, the elements of which are inverse of each other. Therefore the knowledge of the first $M$ eigenvalues or Lyapounov exponents contains the whole information.

The most interesting exponent, which is the smallest positive one, has be studied numerically and was shown to be in agreement with scaling concepts [8, 9]. More recently, the $M$ positive Lyapounov exponents have been investigated. They converge towards a limiting distribution as the width $M$ increases [11].

In order to use the results of section 2 , we need first to find the eigenvalues of the matrix $A$.

Because the system is finite across the strip, the transverse wave vector $q$ is quantized. The $k$-th eigenvalue $\lambda$ of the matrix $A$ is then given by

$$
\lambda_{k}+\lambda_{k}^{-1}=E-2 \cos q_{k} \text {. }
$$

For $t=1$ (periodic boundary conditions), one has

$$
q_{k}=\frac{2 \pi}{M} s_{k}
$$

For $t=0$

$$
q_{k}=\frac{\pi}{M+1} s_{k}
$$

when $s_{k}$ is an integer which can take the values 1 , $2, \ldots, M$.

Let us see now how condition (6) can be satisfied. For a given energy $E$, we have to find the permutation $s_{1}, s_{2}, \ldots, s_{M}$ of the integers $1,2, \ldots, M$ such that $\left|\lambda_{1}\right|>\left|\lambda_{2}\right|>\cdots>\left|\lambda_{M}\right|$. This condition will satisfy automatically (6), since (31) implies that $\lambda_{2 M+1-j}=1 / \lambda_{j}$.

For periodic boundary conditions and at any energy $E$, the eigenvalue corresponding to $s_{k}$ is always the same as the eigenvalue corresponding to $s_{k^{\prime}}=M+1-s_{k}$. Therefore there is no choice of the $s_{k}$ which satisfies condition (6).

For rigid boundary conditions, there are only some special energies $E$ (vertical line in the complex plane of $E$ and a part of the real axis) for which two eigenvalues have the same modulus. For all other energies there is a choice of the $s_{k}$ which satisfies condition (6). For real energies outside the band, $\left(|-E|>2+2 \cos \frac{\pi}{M+1}\right)$, this choice is simple

$$
\text { for } \begin{aligned}
E>2+2 \cos \frac{\pi}{M+1} \\
\qquad s_{k}=M+1-k \quad 1 \leqslant k \leqslant M
\end{aligned}
$$

for $E<-2-2 \cos \frac{\pi}{M+1} \quad s_{k}=k \quad 1 \leqslant k \leqslant M$.

One should notice that in the band

$$
-2-2 \cos \frac{\pi}{M+1}<E<2+2 \cos \frac{\pi}{M+1}
$$

there are always pairs of solutions of (31) which have a modulus 1 . Thus condition (6) can never be satisfied in the band.

In the following, we shall limit ourselves to the case of rigid boundary conditions and to energies $E$ which are either real with $|E|>4$ or in regions of the complex plane where all the eigenvalues of $A$ have different moduli.

The moduli of the eigenvalues being arranged in descending magnitudes for the considered energy $\left(\left|\lambda_{1}\right|>\left|\lambda_{2}\right|>\cdots>\left|\lambda_{k}\right|>\cdots\right)$, we will denote $q(k)$ the transverse wave vector corresponding to the eigenvalue $\lambda_{k}$.

In the basis where $A$ is diagonal, the random matrix $B_{\alpha}$ has the following form

$$
B_{\alpha}=\left(\begin{array}{rr}
\Delta X^{-1} \mathbb{V}_{\alpha} X \Lambda & \Delta X^{-1} \mathbb{V}_{\alpha} X \Lambda^{-1} \\
-\Delta X^{-1} \mathbb{V}_{\alpha} X \Lambda & -\Delta X^{-1} \mathbb{V}_{\alpha} X \Lambda^{-1}
\end{array}\right)
$$

where $\Lambda, X$ and $\Delta$ are $M \times M$ matrices defined by

$$
\begin{gathered}
\Lambda=\left(\begin{array}{ccc}
\lambda_{1} & & \\
& \lambda_{2} & 0 \\
0 & \ddots & \\
& & \lambda_{M}
\end{array}\right) \\
X_{i j}=\sin (i q(j)) \\
\Delta=\left(\Lambda-\Lambda^{-1}\right)^{-1} .
\end{gathered}
$$

Note that the inverse $X^{-1}$ of $X$ is given by

$$
\left(X^{-1}\right)_{i j}=\frac{2}{M+1} \sin (q(i) j) \text {. }
$$

We can now use formula (7) for the disordered strip. In general, our expressions give complex numbers, the real part of which are the inverse localisation lengths. Let us note by $\bar{V}, \overline{V^{2}}, \overline{V^{3}} \ldots$ the moments of the distribution of the diagonal random elements of $V_{\alpha}$. For symmetric distribution, all the odd moments vanish and formula 7 is reduced to

$$
\begin{aligned}
\sum_{j=1}^{p} \gamma_{j}=\sum_{j=1}^{p} \log \lambda_{j}-\frac{\mu^{2}}{2} C_{p}-\frac{\mu^{4}}{4} Q_{1, p}- \\
-\frac{\mu^{4}}{2} Q_{2, p}+\mu^{4} Q_{3, p}+0\left(\mu^{6}\right)
\end{aligned}
$$

where $C_{p}, Q_{1, p}, Q_{2, p}$ and $Q_{3, p}$ are related to the matrices $\Lambda$ and $X$. Let us define

$$
\begin{aligned}
d_{j} & =\left(\lambda_{j}-\lambda_{j}^{-1}\right)^{-1} \\
D_{i j k l} & =d_{i} d_{j} d_{k} d_{l}
\end{aligned}
$$




$$
\begin{gathered}
T_{i j}=\sum_{s=1}^{M}\left(X_{s j} X_{s i}\right)^{2} \\
W_{i j k l}=\sum_{s=1}^{M}\left(X_{s i} X_{s j} X_{s k} X_{s l}\right)^{2} \\
Y_{i j k l}=\sum_{s=1}^{M} \sum_{t=1}^{M} X_{i s} X_{s j}^{2} X_{s k} X_{k t} X_{t l}^{2} X_{t i}
\end{gathered}
$$

$$
Z_{i j k l}=\sum_{s=1}^{M} \sum_{t=1}^{M} X_{i s} X_{s j} X_{k s} X_{s l} X_{j t} X_{t k} X_{l t} X_{t i}
$$

which yield

$$
C_{p}=4 \frac{\overline{V^{2}}}{(M+1)^{2}} \sum_{i, j=1}^{p} T_{i j} d_{i} d_{j}
$$

$$
\begin{gathered}
Q_{1, p}=\frac{16}{(M+1)^{4}} \sum_{i, j, k, l=1}^{p} D_{i j k l}\left[\left(\overline{V^{4}}-3\left(\overline{V^{2}}\right)^{2}\right) W_{i j k l}+\left(\overline{V^{2}}\right)^{2}\left(2 Y_{i j k l}+Z_{i j k l}\right)\right] \\
Q_{2, p}=\frac{16}{(M+1)^{4}}\left(\overline{V^{2}}\right)^{2} \sum_{i, j=1}^{p}\left[\sum_{k, l=p+1}^{M} \frac{\lambda_{k} \lambda_{l}}{\lambda_{i} \lambda_{j}-\lambda_{k} \lambda_{l}} D_{i k j l} Z_{i k j l}-\right. \\
\left.-2 \sum_{k=1}^{M} \sum_{l=p+1}^{M} D_{i k j l} Z_{i k j l} \frac{\lambda_{k}^{-1} \lambda_{l}}{\lambda_{i} \lambda_{j}-\lambda_{k}^{-1} \lambda_{l}}+\sum_{k, l=1}^{M} \frac{D_{i k j l} Z_{i k j l}}{\lambda_{i} \lambda_{j} \lambda_{k} \lambda_{l}-1}\right] \\
Q_{3, p}=\frac{16}{(M+1)^{4}} \bar{V}^{2} \sum_{i, j, k=1}^{p}\left[\sum_{r=p+1}^{M} D_{i j r k} Y_{i r j k} \frac{\lambda_{r}}{\lambda_{i}-\lambda_{r}}-\sum_{r=1}^{M} \frac{D_{i j r k} Y_{i j r k}}{\lambda_{i} \lambda_{r}-1}\right] .
\end{gathered}
$$

It is probably possible to simplify the expressions (40), (41) and (42). However, we did not succeed in writing them in a much more compact form. A difficulty in doing so is that for complex energies, $s_{k}$ or the $q(k)$ do not depend on $k$ in a simple way. Even without simplifying these expressions, they are explicit enough to draw curves on the computer.

When the width $M$ increases, one can compute the weak disorder expansion of the density of Lyapounov exponents [11-14]. Let us take the example of a real energy $E$ outside the band $(|E|>4)$. The $s_{k}$ are then given by (34).

If we limit ourselves to the second order in $\mu$, one finds that

$\gamma_{i}=K_{i}-\mu^{2} \frac{\overline{V^{2}}}{M+1} \frac{1}{\sinh K_{i}}\left[a_{i}+\frac{1}{4} \sum_{j=1}^{i} \frac{1}{\sinh K_{j}}\right]$

where $\grave{a}_{i}$ and $K_{i}$ are given by

$a_{i}=-\frac{1}{16} \frac{1}{\sinh K_{i}}$

$$
\text { if } i<\frac{M+1}{2}
$$

$a_{i}=-\frac{1}{16} \frac{1}{\sinh K_{i}}+\frac{1}{8 \sinh K_{M+1-i}} \quad$ if $\quad i>\frac{M+1}{2}$

and

$$
2 \cosh K_{i}=|E|+2 \cos \frac{\pi i}{M+1} .
$$

For $i=\frac{M+1}{2}$ ( $M$ odd $),(44)$ is slightly more complicated.

In the limit $M \rightarrow \infty$, we see that $\gamma_{i}$ becomes a function of $q=\frac{i \pi}{M+1}$ only, the contribution of $a_{i}$ disappears and the sum becomes an integral

$\gamma_{i}=K(q)-\frac{\mu^{2} \overline{V^{2}}}{4 \pi} \frac{1}{\sinh (K(q))} \int_{0}^{q} \frac{\mathrm{d} q^{\prime}}{\sinh K\left(q^{\prime}\right)}$

where $K(q)$ is given by

$$
2 \cosh K(q)=|E|+2 \cos q .
$$

Formula (46) shows how in the limit $M \rightarrow \infty$ the distribution of Lyapounov exponents is modified by the disorder.

Such an asymptotic distribution possesses an interesting physical meaning [11]. For a sample of finite longitudinal length $N$, the $j$-channel has a good transmission when $\left(\gamma_{j}\right)^{-1}>N$. Therefore (46) gives the density of active transmission channels [18] which can be related to the conductance [19].

\section{Comparison with numerical simulations.}

For real energies such that $|E|>2+2 \cos \frac{\pi}{M+1}$ and for rigid boundary conditions, we have used formulae (40), (41) and (42) to compute the Lyapounov exponents up to the $4^{\text {th }}$ order in $\mu$. In order to compare with the results of numerical simulations, we have considered a strip of width 3 at energy $E=4$ and a flat distribution of the potential $V_{i, j}\left[\rho\left(V_{i, j}\right)=\frac{1}{W}\right.$ for $\left|V_{i, j}\right|<\frac{W}{2}$ and $=0$ for $\left.\left|V_{i, j}\right|>\frac{W}{2}\right]$. In figure 1 , the points represent the Lyapounov exponents estimated by performing the 


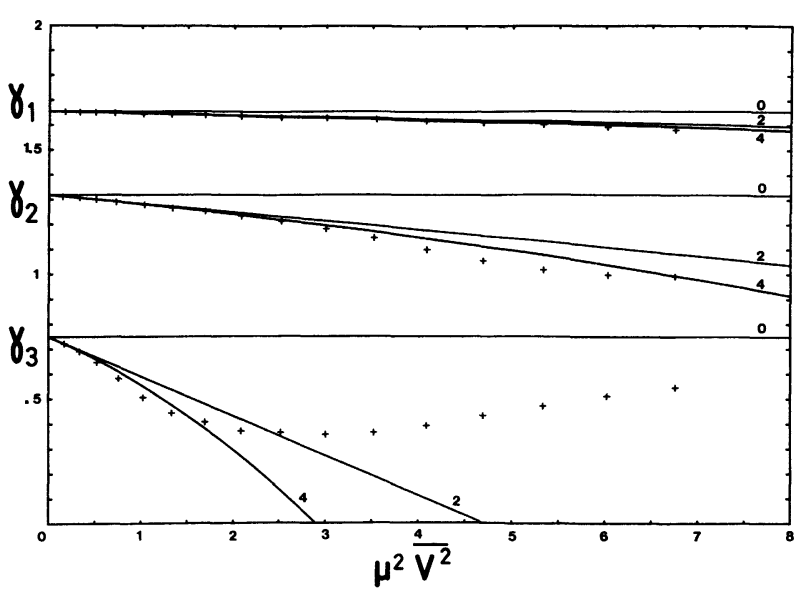

Fig. 1. - Positive Lyapounov exponents as a function of the strength of disorder $\mu^{2} \bar{V}^{2}$ for a strip of width $M=3$ at energy $E=4$. The points $(+)$ represent the results of numerical simulations and the curves the weak disorder expansion up to $0^{\text {th }}$ order $(0), 2^{\text {nd }}$ order (2) and $4^{\text {th }}$ order (4).

product of 50000 random matrices. The curves represent the predictions of formulae (40), (41) and (42) truncated at the $0^{\text {th }}$ order, the $2^{\text {nd }}$ order and the $4^{\text {th }}$ order in $\mu$. We see that as one increases the order in the expansion, the agreement with the numerical results becomes better and better.

For real energies $|E|<2+2 \cos \frac{\pi}{M+1}$, one cannot use the expansion because the condition (6), is not satisfied. One can then add an imaginary part $\varepsilon$ to the energy which becomes complex

$$
E=E_{0}+i \varepsilon
$$

Then the condition is true for all energies $E_{0}$ except a few points such that

$$
E_{0}-2 \cos \frac{q(i)}{M+1}= \pm\left(E_{0}-2 \cos \frac{q(j)}{M+1}\right)
$$

which is equivalent to $\left|\lambda_{i}\right|=\left|\lambda_{j}\right|$. This condition gives vertical lines in the complex plane of $E$.

For the example of a strip of width $M=3$, $q(i)$ and $q(j)$ can take only the values $\frac{\pi}{4}, \frac{\pi}{2}$ and $\frac{3 \pi}{4}$ and therefore (49) gives $E_{0}=0, \pm \frac{\sqrt{2}}{2}$.

In figure 2, we show the Lyapounov exponents for zero disorder as a function of $E_{0}$ for a rather large value of $\varepsilon(\varepsilon=0.5)$ and for a strip of width $M=3$. We see that the condition (6) $\left(\left|\lambda_{1}\right|>\right.$ $\left.\left|\lambda_{2}\right|>\left|\lambda_{3}\right|\right)$ is satisfied for all $E_{0}$ except at the points $E_{0}=0, \pm \frac{\sqrt{2}}{2}$.

In figure 3 , we show as a function of $E_{0}$ the difference between the results obtained numerically by multiplying 50000 random matrices and the expressions (40), (41), (42) truncated at the $0^{\text {th }}$, the

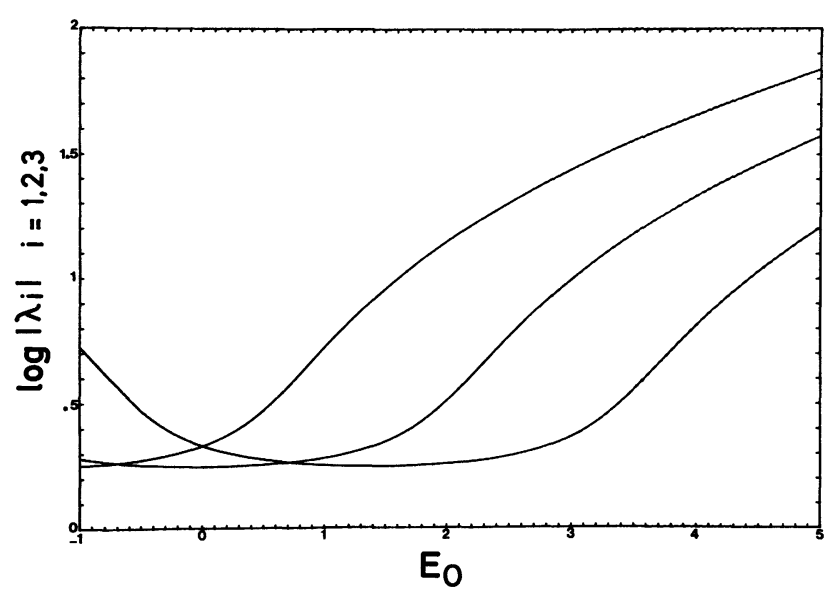

Fig. 2. $-\log \left|\lambda_{i}\right| \quad(i=1,2,3)$ for a strip without disorder as a function of $E_{0} . M=3, \varepsilon=0.5$. Condition (6) is satisfied except for $E_{0}=0$ and $E_{0}= \pm \sqrt{2} / 2$.

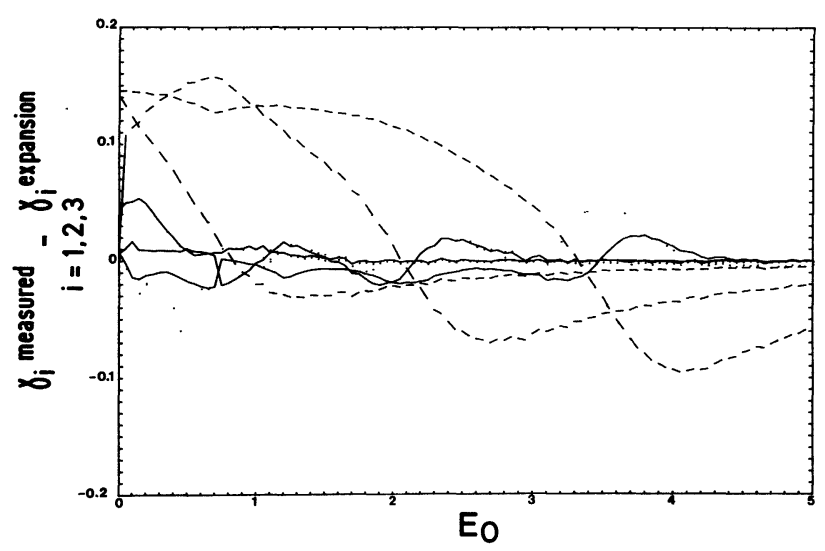

Fig. 3. - Difference between the positive Lyapounov exponents computed by numerical simulations and the non-degenerate expansion up to $0^{\text {th }}$ order $(---)$, up to $2^{\text {nd }}$ order $(\ldots)$ and up to $4^{\text {th }}$ order $(-)$ as a function of $E_{0}$ for $M=3, \varepsilon=0.5$ and $\mu^{2} \overline{V^{2}}=1$.

$2^{\text {nd }}$ and the $4^{\text {th }}$ order. The strip has a width $M=3$, the flat distribution of the $V_{i j}$ is such tha: $\overline{V_{i j}^{2}}=1$, and $\varepsilon=0.5$. We see that the agreement is usually improved by increasing the order of the expansion. We do not see clearly that at the special values $E_{0}=0, \pm \frac{\sqrt{2}}{2}$ the expansion is not valid. Several reasons might be responsible for it : too bad statistics, too strong disorder or a value of $\varepsilon$ too small. Even if the agreement is worse than for $|E|>4$, it remains satisfactory.

In order to see what happens inside the band, we show in figure 4 the Lyapounov exponents computed from formulas (40-41 and 42) for a small value of $\varepsilon$ $(\varepsilon=0.01)$. We see that our expansion blows up at several energies : first the energies which correspond to the band edges of each $j$ channel (these energies are given by $|E-2 \cos q(j)|=2$ i.e. $\left.\lambda_{j}= \pm 1\right)$. In 


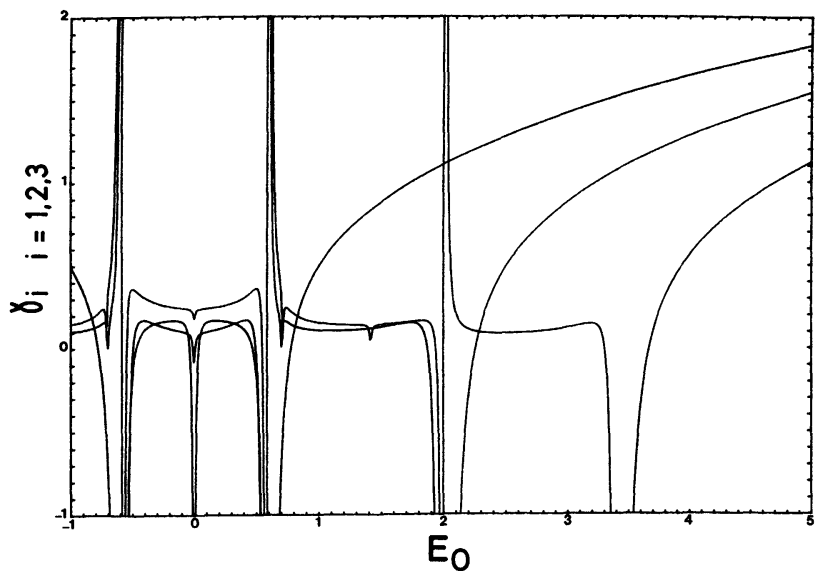

Fig. 4. - Non degenerate perturbation expansion up to the fourth order (formula (24)) of the positive Lyapounov exponents of a strip of width $M=3$ as a function of the energy $E_{0}$ inside the band for $\varepsilon=0.01$ and $\mu^{2} \bar{V}^{2}=1$.

addition, one can see that the fourth order term yields new divergences at the band centre of each $j$ channel $(E=2 \cos q(j))$ where $\lambda_{j}=\mathrm{e}^{i \pi / 2}$ and more generally at all energies where $\lambda_{i}=\lambda_{j}$ or $\lambda_{i} \lambda_{j}=\lambda_{k} \lambda_{r}$ see formula (7). The number of these singular energies will increase with the width of the strip and with the order in the weak disorder expansion. These singular behaviors are already present in the case of the chain $(M=1)$ at the band edge and at the band centre. For the chain one knows how to derive the right weak disorder expansion in the neighbourhood of these special energies [4-7]. We think that it should be possible to generalize these approaches to the case of strips.

\section{Conclusion.}

In this work, we have obtained the weak disorder expansion of the Lyapounov exponents of a product of random matrices. When condition (6) is satisfied, the agreement between numerical simulations and our expansion is satisfactory for the problem of localisation on strips.

So our expansion can be used to compute the Lyapounov exponents for the localisation problem on disordered strips for energies outside the band of the non-disordered strip or for complex energies. For energies inside the band of the non-disordered strip, condition (6) is not satisfied and our expansion is no longer valid. The presence of small denominators in formula (7) shows that the degenerate case (the case for which (6) is not satisfied) cannot be in general treated by a simple extrapolation of formula (7).

We think that it would be very interesting to generalize our approach to degenerate cases, i.e. to cases for which condition (6) is not satisfied and also to cases where the matrix A cannot be diagonalized. This would allow to compute the Lyapounov exponents inside the band and would give a better understanding of the scaling [8] of the Lyapounov exponents with the strip width. Then, by looking at the $M$ dependence of the Lyapounov exponents [8, 9], one would have a new analytical way of understanding two-dimensional localization.

\section{Acknowledgments.}

K. Mecheri is grateful to the Service de Physique du Solide et de Résonance Magnétique of the CENSaclay for its hospitality.

\section{References}

[1] Dyson, F. J., Phys. Rev. 92 (1953) 1331.

[2] Alexander, S., Bernasconi, J., SCHneider, W. R. and Orbach, R., Rev. Mod. Phys. 53 (1981) 175.

[3] Derrida, B., Lecture Notes in Physics 226 (Springer-Verlag) 1985, p. 276.

[4] Kappus, M. and Wegner, F., Z. Phys. B 45 (1981) 15.

[5] Derrida, B. and Gardner, E., J. Physique 45 (1984) 1283.

[6] Lambert, C. J., Phys. Rev. B 29 (1984) 1091.

[7] Mielke, A. and Wegner, F., Z. Phys. B 62 (1985) 1.

[8] Pichard, J. L. and Sarma, G., J. Phys. C 14 (1981) L127.

[9] Pichard, J. L. and SARMa, G., J. Phys. C 14 (1981) L617.

[10] Lacroix, J., Ann. Inst. H. Poincaré 40A (1984) 97.
[11] Pichard, J. L. and ANDRÉ, G., Europhys. Lett. 2 (6) (1986) 477.

[12] Ruelle, D., Comm. Math. Phys. 87 (1982) 287.

[13] Newman, C. M., Comm. Math. Phys. 103 (1986) 121.

[14] Paladin, G. and Vulpiani, J. Phys. A 19 (1986) 1881.

[15] Livi, R., Politi, A. and Ruffo, S., J. Phys. A : Math. Gen. 19 (1986) 2033.

[16] Eckmann, J. P. and Ruelle, D., Rev. Mod. Phys. 57 (1985) 617.

[17] Benettin, G., Galgani, L., Giorgilli, A., StrelCYN, J. M., C. R. Heb. Séan. Acad. Sci. 286A (1978) 431.

[18] IMry, Y., Europhys. Lett. 1 (5) (1986) 249.

[19] BÜTTIKER, M., IMRY, Y., LANDAUER, R. and PINHAS, S., Phys. Rev. B 31 (1985) 6207. 